\title{
Generalized (anti) Yetter-Drinfeld modules as components of a braided T-category
}

\author{
Florin Panaite* \\ Institute of Mathematics of the Romanian Academy \\ PO-Box 1-764, RO-014700 Bucharest, Romania \\ e-mail: Florin.Panaite@imar.ro \\ Mihai D. Staic ${ }^{\dagger}$ \\ SUNY at Buffalo \\ Amherst, NY 14260-2900, USA \\ e-mail: mdstaic@buffalo.edu
}

\begin{abstract}
If $H$ is a Hopf algebra with bijective antipode and $\alpha, \beta \in A u t_{H o p f}(H)$, we introduce a category ${ }_{H} \mathcal{Y D}^{H}(\alpha, \beta)$, generalizing both Yetter-Drinfeld modules and anti-Yetter-Drinfeld modules. We construct a braided T-category $\mathcal{Y} \mathcal{D}(H)$ having all the categories ${ }_{H} \mathcal{Y D}^{H}(\alpha, \beta)$ as components, which if $H$ is finite dimensional coincides with the representations of a certain quasitriangular T-coalgebra $D T(H)$ that we construct. We also prove that if $(\alpha, \beta)$ admits a so-called pair in involution, then ${ }_{H} \mathcal{Y} \mathcal{D}^{H}(\alpha, \beta)$ is isomorphic to the category of usual YetterDrinfeld modules ${ }_{H} \mathcal{Y} \mathcal{D}^{H}$.
\end{abstract}

\section{Introduction}

Let $H$ be a Hopf algebra with bijective antipode $S$ and $\alpha, \beta \in A u t_{H o p f}(H)$. We introduce the concept of an $(\alpha, \beta)$-Yetter-Drinfeld module, as being a left $H$-module right $H$-comodule $M$ with the following compatibility condition:

$$
(h \cdot m)_{(0)} \otimes(h \cdot m)_{(1)}=h_{2} \cdot m_{(0)} \otimes \beta\left(h_{3}\right) m_{(1)} \alpha\left(S^{-1}\left(h_{1}\right)\right) .
$$

This concept is a generalization of three kinds of objects appeared in the literature. Namely, for $\alpha=\beta=i d_{H}$, we obtain the usual Yetter-Drinfeld modules; for $\alpha=S^{2}, \beta=i d_{H}$, we obtain the so-called anti-Yetter-Drinfeld modules, introduced in [7, 8], 10] as coefficients for the cyclic cohomology of Hopf algebras defined by Connes and Moscovici in [5], [6] finally, an $\left(i d_{H}, \beta\right)$ Yetter-Drinfeld module is a generalization of the object $H_{\beta}$ defined in [4, which has the property that, if $H$ is finite dimensional, then the map $\beta \mapsto \operatorname{End}\left(H_{\beta}\right)$ gives a group anti-homomorphism from $A_{u t} t_{\text {Hopf }}(H)$ to the Brauer group of $H$.

It is natural to expect that $(\alpha, \beta)$-Yetter-Drinfeld modules have some properties resembling the

\footnotetext{
${ }^{*}$ Research partially supported by the programme CERES of the Romanian Ministry of Education and Research, contract no. 4-147/2004.

${ }^{\dagger}$ Permanent address: Institute of Mathematics of the Romanian Academy, PO-Box 1-764, RO-014700 Bucharest, Romania.
} 
ones of the three kinds of objects we mentioned. We will see some of these properties in this paper (others will be given in a subsequent one), namely the ones directed to our main aim here, which is the following: if we denote by ${ }_{H} \mathcal{Y D}^{H}(\alpha, \beta)$ the category of $(\alpha, \beta)$-Yetter-Drinfeld modules and we define $\mathcal{Y} \mathcal{D}(H)$ as the disjoint union of all these categories, then we can organize $\mathcal{Y} \mathcal{D}(H)$ as a braided T-category (or braided crossed group-category, in the original terminology of Turaev, see [16]) over the group $G=\operatorname{Aut}_{\text {Hopf }}(H) \times \operatorname{Aut}_{H o p f}(H)$ with multiplication $(\alpha, \beta) *(\gamma, \delta)=$ $\left(\alpha \gamma, \delta \gamma^{-1} \beta \gamma\right)$. We also prove that the subcategory $\mathcal{Y} \mathcal{D}(H)_{f d}$ consisting of finite dimensional objects has left and right dualities, and that, if $H$ is finite dimensional, then $\mathcal{Y} \mathcal{D}(H)$ coincides with the representations of a certain quasitriangular T-coalgebra $D T(H)$ that we construct. Our second aim is to prove that, if $\alpha, \beta \in \operatorname{Aut}_{\text {Hopf }}(H)$ such that there exists a so-called pair in involution $(f, g)$ corresponding to $(\alpha, \beta)$, then ${ }_{H} \mathcal{Y} \mathcal{D}^{H}(\alpha, \beta)$ is isomorphic to ${ }_{H} \mathcal{Y D}^{H}$. This result is independent on the theory concerning $\mathcal{Y} \mathcal{D}(H)$, but we can give it a very short proof using the results obtained during the construction of $\mathcal{Y} \mathcal{D}(H)$.

\section{Preliminaries}

We work over a ground field $k$. All algebras, linear spaces, etc. will be over $k$; unadorned $\otimes$ means $\otimes_{k}$. Unless otherwise stated, $H$ will denote a Hopf algebra with bijective antipode $S$. We will use the versions of Sweedler's sigma notation: $\Delta(h)=h_{1} \otimes h_{2}$ or $\Delta(h)=h_{(1)} \otimes h_{(2)}$. For unexplained concepts and notation about Hopf algebras we refer to [11], 12], [13], [15]. By $\alpha, \beta, \gamma \ldots$ we will usually denote Hopf automorphisms of $H$.

Let $A$ be an $H$-bicomodule algebra, with comodule structures $A \rightarrow A \otimes H, a \mapsto a_{<0>} \otimes a_{<1>}$ and $A \rightarrow H \otimes A, a \mapsto a_{[-1]} \otimes a_{[0]}$, and denote, for $a \in A$,

$$
a_{\{-1\}} \otimes a_{\{0\}} \otimes a_{\{1\}}=a_{<0>_{[-1]}} \otimes a_{<0>[0]} \otimes a_{<1>}=a_{[-1]} \otimes a_{[0]_{<0>}} \otimes a_{[0]_{<1>}}
$$

as an element in $H \otimes A \otimes H$. We can consider the Yetter-Drinfeld datum $(H, A, H)$ as in [3] (the second $H$ is regarded as an $H$-bimodule coalgebra), and the Yetter-Drinfeld category ${ }_{A} \mathcal{Y} \mathcal{D}(H)^{H}$, whose objects are $k$-modules $M$ endowed with a left $A$-action (denoted by $a \otimes m \mapsto a \cdot m$ ) and a right $H$-coaction (denoted by $m \mapsto m_{(0)} \otimes m_{(1)}$ ) satisfying the equivalent compatibility conditions

$$
\begin{aligned}
& (a \cdot m)_{(0)} \otimes(a \cdot m)_{(1)}=a_{\{0\}} \cdot m_{(0)} \otimes a_{\{1\}} m_{(1)} S^{-1}\left(a_{\{-1\}}\right), \\
& a_{<0>} \cdot m_{(0)} \otimes a_{<1>} m_{(1)}=\left(a_{[0]} \cdot m\right)_{(0)} \otimes\left(a_{[0]} \cdot m\right)_{(1)} a_{[-1]},
\end{aligned}
$$

for all $a \in A$ and $m \in M$.

Recall now from [9] the construction of the (left) diagonal crossed product $H^{*} \bowtie A$, which is an associative algebra constructed on $H^{*} \otimes A$, with multiplication given by

$$
(p \bowtie a)(q \bowtie b)=p\left(a_{\{-1\}} \rightarrow q<S^{-1}\left(a_{\{1\}}\right)\right) \bowtie a_{\{0\}} b,
$$

for all $a, b \in A$ and $p, q \in H^{*}$, and with unit $\varepsilon_{H} \bowtie 1_{A}$. Here $\rightarrow$ and $\angle$ are the regular actions of $H$ on $H^{*}$ given by $(h \rightarrow p)(l)=p(l h)$ and $(p \leftarrow h)(l)=p(h l)$ for all $h, l \in H$ and $p \in H^{*}$.

If $H$ is finite dimensional, we can consider the Drinfeld double $D(H)$, which is a quasitriangular Hopf algebra realized on $H^{*} \otimes H$; its coalgebra structure is $H^{* c o p} \otimes H$ and the algebra structure is just $H^{*} \bowtie H$, that is

$$
(p \bowtie h)(q \bowtie l)=p\left(h_{1} \rightarrow q<S^{-1}\left(h_{3}\right)\right) \bowtie h_{2} l,
$$


for all $p, q \in H^{*}$ and $h, l \in H$.

The diagonal crossed product $H^{*} \bowtie A$ becomes a $D(H)$-bicomodule algebra, with structures

$$
\begin{aligned}
& H^{*} \bowtie A \rightarrow\left(H^{*} \bowtie A\right) \otimes D(H), p \bowtie a \mapsto\left(p_{2} \bowtie a_{<0>}\right) \otimes\left(p_{1} \otimes a_{<1>}\right), \\
& H^{*} \bowtie A \rightarrow D(H) \otimes\left(H^{*} \bowtie A\right), p \bowtie a \mapsto\left(p_{2} \otimes a_{[-1]}\right) \otimes\left(p_{1} \bowtie a_{[0]}\right),
\end{aligned}
$$

for all $p \in H^{*}$ and $a \in A$, see $[9$.

In the case when $H$ is finite dimensional, by results in [1, 3] it follows that the category ${ }_{A} \mathcal{Y D}(H)^{H}$ is isomorphic to the category ${ }_{H^{*} \bowtie A} \mathcal{M}$ of left modules over $H^{*} \bowtie A$.

\section{$2(\alpha, \beta)$-Yetter-Drinfeld modules}

Definition 2.1 Let $\alpha, \beta \in$ Aut $_{\text {Hopf }}(H)$. An $(\alpha, \beta)$-Yetter-Drinfeld module over $H$ is a vector space $M$, such that $M$ is a left $H$-module (with notation $h \otimes m \mapsto h \cdot m$ ) and a right $H$-comodule (with notation $M \rightarrow M \otimes H, m \mapsto m_{(0)} \otimes m_{(1)}$ ) with the following compatibility condition:

$$
(h \cdot m)_{(0)} \otimes(h \cdot m)_{(1)}=h_{2} \cdot m_{(0)} \otimes \beta\left(h_{3}\right) m_{(1)} \alpha\left(S^{-1}\left(h_{1}\right)\right)
$$

for all $h \in H$ and $m \in M$. We denote by ${ }_{H} \mathcal{Y D}^{H}(\alpha, \beta)$ the category of $(\alpha, \beta)$-Yetter-Drinfeld modules, morphisms being the $H$-linear $H$-colinear maps.

Remark 2.2 As for usual Yetter-Drinfeld modules, one can see that (2.1) is equivalent to

$$
h_{1} \cdot m_{(0)} \otimes \beta\left(h_{2}\right) m_{(1)}=\left(h_{2} \cdot m\right)_{(0)} \otimes\left(h_{2} \cdot m\right)_{(1)} \alpha\left(h_{1}\right) .
$$

Example 2.3 For $\alpha=\beta=i d_{H}$, we have ${ }_{H} \mathcal{Y D}^{H}(i d, i d)={ }_{H} \mathcal{Y D}^{H}$, the usual category of (leftright) Yetter-Drinfeld modules.

Example 2.4 For $\alpha=S^{2}, \beta=i d_{H}$, the compatibility condition (2.1) becomes

$$
(h \cdot m)_{(0)} \otimes(h \cdot m)_{(1)}=h_{2} \cdot m_{(0)} \otimes h_{3} m_{(1)} S\left(h_{1}\right),
$$

hence ${ }_{H} \mathcal{Y D}^{H}\left(S^{2}, i d\right)$ is the category of anti-Yetter-Drinfeld modules defined in [7], [8], [10].

Example 2.5 For $\beta \in$ Aut $_{\text {Hopf }}(H)$, define $H_{\beta}$ as in [4], that is $H_{\beta}=H$, with regular right $H$-comodule structure and left $H$-module structure given by $h \cdot h^{\prime}=\beta\left(h_{2}\right) h^{\prime} S^{-1}\left(h_{1}\right)$, for all $h, h^{\prime} \in H$. It was noticed in [4] that $H_{\beta}$ satisfies a certain compatibility condition, which actually says that $H_{\beta} \in{ }_{H} \mathcal{Y D}^{H}(i d, \beta)$. More generally, if $\alpha, \beta \in A u t_{H o p f}(H)$, define $H_{\alpha, \beta}$ as follows: $H_{\alpha, \beta}=H$, with regular right $H$-comodule structure and left $H$-module structure given by $h \cdot h^{\prime}=$ $\beta\left(h_{2}\right) h^{\prime} \alpha\left(S^{-1}\left(h_{1}\right)\right)$, for $h, h^{\prime} \in H$. Then one can check that $H_{\alpha, \beta} \in{ }_{H} \mathcal{Y D}^{H}(\alpha, \beta)$.

Example 2.6 Take $l$ an integer and define $\alpha_{l}=S^{2 l} \in$ Aut $_{H o p f}(H)$. The compatibility in ${ }_{H} \mathcal{Y} \mathcal{D}^{H}\left(S^{2 l}, i d\right)$ becomes

$$
(h \cdot m)_{(0)} \otimes(h \cdot m)_{(1)}=h_{2} \cdot m_{(0)} \otimes h_{3} m_{(1)} S^{2 l-1}\left(h_{1}\right) .
$$

An object in ${ }_{H} \mathcal{Y D}^{H}\left(S^{2 l}, i d\right)$ will be called an $l-\mathcal{Y D}$-module. Hence, a $0-\mathcal{Y} \mathcal{D}$-module is a Yetter-Drinfeld module and a $1-\mathcal{Y D}$-module is an anti-Yetter-Drinfeld module. The right-left version of $l-\mathcal{Y} \mathcal{D}$-modules has been introduced in [14]. 
Example 2.7 Let $\alpha, \beta \in$ Aut $_{\text {Hopf }}(H)$ and assume that there exist an algebra map $f: H \rightarrow k$ and a group-like element $g \in H$ such that

$$
\alpha(h)=g^{-1} f\left(h_{1}\right) \beta\left(h_{2}\right) f\left(S\left(h_{3}\right)\right) g, \quad \forall h \in H .
$$

Then one can check that $k \in{ }_{H} \mathcal{Y D}^{H}(\alpha, \beta)$, with structures $h \cdot 1=f(h)$ and $1 \mapsto 1 \otimes g$. More generally, if $V$ is any vector space, then $V \in{ }_{H} \mathcal{Y} \mathcal{D}^{H}(\alpha, \beta)$, with structures $h \cdot v=f(h) v$ and $v \mapsto v \otimes g$, for all $h \in H$ and $v \in V$.

Definition 2.8 If $\alpha, \beta \in$ Aut $_{\text {Hopf }}(H)$ such that there exist $f, g$ as in Example 2.7, we will say that $(f, g)$ is a pair in involution corresponding to $(\alpha, \beta)$ (in analogy with the concept of modular pair in involution due to Connes and Moscovici) and the $(\alpha, \beta)$-Yetter-Drinfeld modules $k$ and $V$ constructed in Example 2.7 will be denoted by ${ }_{f} k^{g}$ and respectively ${ }_{f} V^{g}$.

As an example, if $\alpha \in A u t_{H o p f}(H)$, then $(\varepsilon, 1)$ is a pair in involution corresponding to $(\alpha, \alpha)$.

Let $\alpha, \beta \in \operatorname{Aut}_{\text {Hopf }}(H)$. We define an $H$-bicomodule algebra $H(\alpha, \beta)$ as follows: $H(\alpha, \beta)=H$ as algebra, with comodule structures

$$
\begin{array}{ll}
H(\alpha, \beta) \rightarrow H \otimes H(\alpha, \beta), & h \mapsto h_{[-1]} \otimes h_{[0]}=\alpha\left(h_{1}\right) \otimes h_{2}, \\
H(\alpha, \beta) \rightarrow H(\alpha, \beta) \otimes H, & h \mapsto h_{<0>} \otimes h_{<1>}=h_{1} \otimes \beta\left(h_{2}\right) .
\end{array}
$$

Then we can consider the Yetter-Drinfeld datum $(H, H(\alpha, \beta), H)$ and the Yetter-Drinfeld modules over it, $H(\alpha, \beta) \mathcal{Y} \mathcal{D}(H)^{H}$.

Proposition $2.9{ }_{H} \mathcal{Y} \mathcal{D}^{H}(\alpha, \beta)={ }_{H(\alpha, \beta)} \mathcal{Y} \mathcal{D}(H)^{H}$.

Proof. It is easy to see that the compatibility conditions for the two categories are the same.

In particular, the category of anti-Yetter-Drinfeld modules coincides with $H_{\left(S^{2}, i d\right)} \mathcal{Y D}(H)^{H}$, which improves the remark in [7] that anti-Yetter-Drinfeld modules are entwined modules.

Consider now the diagonal crossed product $A(\alpha, \beta)=H^{*} \bowtie H(\alpha, \beta)$, whose multiplication is

$$
(p \bowtie h)(q \bowtie l)=p\left(\alpha\left(h_{1}\right) \rightarrow q<S^{-1}\left(\beta\left(h_{3}\right)\right)\right) \bowtie h_{2} l,
$$

for all $p, q \in H^{*}$ and $h, l \in H$. For $\alpha=\beta=i d$ we get $A(i d, i d)=D(H)$; for $\alpha=S^{2}$ and $\beta=i d$, the multiplication in $A\left(S^{2}, i d\right)$ is

$$
(p \bowtie h)(q \bowtie l)=p\left(S^{2}\left(h_{1}\right) \rightarrow q \leftarrow S^{-1}\left(h_{3}\right)\right) \bowtie h_{2} l,
$$

hence $A\left(S^{2}, i d\right)$ coincides with the algebra $A(H)$ defined in [7].

Assume now that $H$ is finite dimensional; then $A(\alpha, \beta)$ becomes a $D(H)$-bicomodule algebra, with structures

$$
\begin{array}{ll}
H^{*} \bowtie H(\alpha, \beta) \rightarrow\left(H^{*} \bowtie H(\alpha, \beta)\right) \otimes D(H), & p \bowtie h \mapsto\left(p_{2} \bowtie h_{1}\right) \otimes\left(p_{1} \otimes \beta\left(h_{2}\right)\right), \\
H^{*} \bowtie H(\alpha, \beta) \rightarrow D(H) \otimes\left(H^{*} \bowtie H(\alpha, \beta)\right), & p \bowtie h \mapsto\left(p_{2} \otimes \alpha\left(h_{1}\right)\right) \otimes\left(p_{1} \bowtie h_{2}\right) .
\end{array}
$$

In particular, $A(H)$ becomes a $D(H)$-bicomodule algebra, improving the remark in [7] that $A(H)$ is a right $D(H)$-comodule algebra. Since $H$ is finite dimensional, we have an isomorphism of categories $H_{(\alpha, \beta)} \mathcal{Y \mathcal { D } ( H ) ^ { H } \simeq { } _ { H ^ { * } \bowtie H ( \alpha , \beta ) } \mathcal { M } \text { , hence } { } _ { H } \mathcal { Y } \mathcal { D } ^ { H } ( \alpha , \beta ) \simeq H ^ { * } \bowtie H ( \alpha , \beta )} \mathcal{M}$ (for $\alpha=S^{2}$, 
$\beta=i d$ we recover the result in [7] that the category of anti-Yetter-Drinfeld modules is isomorphic

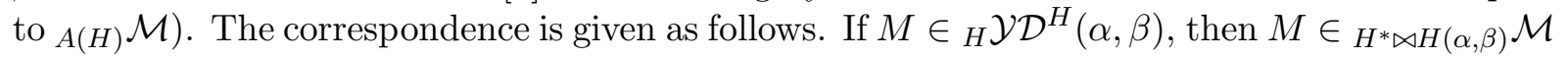
with structure

$$
(p \bowtie h) \cdot m=p\left((h \cdot m)_{(1)}\right)(h \cdot m)_{(0)} .
$$

Conversely, if $M \in{ }_{H^{*} \bowtie H(\alpha, \beta)} \mathcal{M}$, then $M \in{ }_{H} \mathcal{Y D}^{H}(\alpha, \beta)$ with structures

$$
\begin{aligned}
& h \cdot m=(\varepsilon \bowtie h) \cdot m, \\
& m \mapsto m_{(0)} \otimes m_{(1)}=\left(e^{i} \bowtie 1\right) \cdot m \otimes e_{i},
\end{aligned}
$$

where $\left\{e_{i}\right\},\left\{e^{i}\right\}$ are dual bases in $H$ and $H^{*}$.

\section{$3 \quad$ A braided T-category $\mathcal{Y} \mathcal{D}(H)$}

Let $\alpha, \beta \in A u t_{\text {Hopf }}(H)$ and consider the objects $H_{\alpha}, H_{\beta}$ as in Example 2.5. In [4] was considered the object $M=H_{\alpha} \otimes H_{\beta}$, with the following structures:

$$
\begin{aligned}
& h \cdot(x \otimes y)=h_{1} \cdot x \otimes \alpha\left(h_{2}\right) \cdot y, \\
& x \otimes y \mapsto\left(x_{1} \otimes y_{1}\right) \otimes y_{2} x_{2},
\end{aligned}
$$

for all $h, x, y \in H$, where by . we denoted both the actions of $H$ on $H_{\alpha}$ and $H_{\beta}$ given as in Example 2.5. Then it was noticed in [4] that $M$ satisfies a compatibility condition which says that $M \in{ }_{H} \mathcal{Y} \mathcal{D}^{H}(i d, \beta \alpha)$.

On the other hand, it was noticed in [7] that the tensor product between an anti-Yetter-Drinfeld module and a Yetter-Drinfeld module becomes an anti-Yetter-Drinfeld module.

The next result can be seen as a generalization of both these facts.

Proposition 3.1 If $M \in{ }_{H} \mathcal{Y} \mathcal{D}^{H}(\alpha, \beta), N \in{ }_{H} \mathcal{Y} \mathcal{D}^{H}(\gamma, \delta)$, then $M \otimes N \in{ }_{H} \mathcal{Y D}^{H}\left(\alpha \gamma, \delta \gamma^{-1} \beta \gamma\right)$, with structures:

$$
\begin{aligned}
& h \cdot(m \otimes n)=\gamma\left(h_{1}\right) \cdot m \otimes \gamma^{-1} \beta \gamma\left(h_{2}\right) \cdot n, \\
& m \otimes n \mapsto(m \otimes n)_{(0)} \otimes(m \otimes n)_{(1)}=\left(m_{(0)} \otimes n_{(0)}\right) \otimes n_{(1)} m_{(1)} .
\end{aligned}
$$

Proof. Obviously $M \otimes N$ is a left $H$-module and a right $H$-comodule. We check now the compatibility condition. We compute:

$$
\begin{aligned}
(h & \cdot(m \otimes n))_{(0)} \otimes(h \cdot(m \otimes n))_{(1)} \\
= & \left(\gamma\left(h_{1}\right) \cdot m \otimes \gamma^{-1} \beta \gamma\left(h_{2}\right) \cdot n\right)_{(0)} \otimes\left(\gamma\left(h_{1}\right) \cdot m \otimes \gamma^{-1} \beta \gamma\left(h_{2}\right) \cdot n\right)_{(1)} \\
= & \left(\left(\gamma\left(h_{1}\right) \cdot m\right)_{(0)} \otimes\left(\gamma^{-1} \beta \gamma\left(h_{2}\right) \cdot n\right)_{(0)}\right) \otimes\left(\gamma^{-1} \beta \gamma\left(h_{2}\right) \cdot n\right)_{(1)}\left(\gamma\left(h_{1}\right) \cdot m\right)_{(1)} \\
= & \left(\gamma\left(h_{1}\right)_{2} \cdot m_{(0)} \otimes \gamma^{-1} \beta \gamma\left(h_{2}\right)_{2} \cdot n_{(0)}\right) \otimes \\
& \quad \otimes \delta\left(\gamma^{-1} \beta \gamma\left(h_{2}\right)_{3}\right) n_{(1)} \gamma\left(S^{-1}\left(\gamma^{-1} \beta \gamma\left(h_{2}\right)_{1}\right)\right) \beta\left(\gamma\left(h_{1}\right)_{3}\right) m_{(1)} \alpha\left(S^{-1}\left(\gamma\left(h_{1}\right)_{1}\right)\right) \\
= & \left(\gamma\left(h_{2}\right) \cdot m_{(0)} \otimes \gamma^{-1} \beta \gamma\left(h_{5}\right) \cdot n_{(0)}\right) \otimes \delta \gamma^{-1} \beta \gamma\left(h_{6}\right) n_{(1)} \beta \gamma\left(S^{-1}\left(h_{4}\right)\right) \beta \gamma\left(h_{3}\right) m_{(1)} \alpha\left(S^{-1}\left(\gamma\left(h_{1}\right)\right)\right) \\
= & \left(\gamma\left(h_{2}\right) \cdot m_{(0)} \otimes \gamma^{-1} \beta \gamma\left(h_{3}\right) \cdot n_{(0)}\right) \otimes \delta \gamma^{-1} \beta \gamma\left(h_{4}\right) n_{(1)} m_{(1)} \alpha \gamma\left(S^{-1}\left(h_{1}\right)\right) \\
= & h_{2} \cdot\left(m_{(0)} \otimes n_{(0)}\right) \otimes \delta \gamma^{-1} \beta \gamma\left(h_{3}\right) n_{(1)} m_{(1)} \alpha \gamma\left(S^{-1}\left(h_{1}\right)\right) \\
= & h_{2} \cdot(m \otimes n)_{(0)} \otimes \delta \gamma^{-1} \beta \gamma\left(h_{3}\right)(m \otimes n)_{(1)} \alpha \gamma\left(S^{-1}\left(h_{1}\right)\right),
\end{aligned}
$$


that is $M \otimes N \in{ }_{H} \mathcal{Y} \mathcal{D}^{H}\left(\alpha \gamma, \delta \gamma^{-1} \beta \gamma\right)$.

Note that, if $M \in{ }_{H} \mathcal{Y} \mathcal{D}^{H}(\alpha, \beta), N \in{ }_{H} \mathcal{Y} \mathcal{D}^{H}(\gamma, \delta)$ and $P \in{ }_{H} \mathcal{Y} \mathcal{D}^{H}(\mu, \nu)$, then $(M \otimes N) \otimes P=$ $M \otimes(N \otimes P)$ as objects in ${ }_{H} \mathcal{Y} \mathcal{D}^{H}\left(\alpha \gamma \mu, \nu \mu^{-1} \delta \gamma^{-1} \beta \gamma \mu\right)$.

Denote $G=A u t_{H o p f}(H) \times A u t_{H o p f}(H)$, a group with multiplication

$$
(\alpha, \beta) *(\gamma, \delta)=\left(\alpha \gamma, \delta \gamma^{-1} \beta \gamma\right)
$$

(the unit is $(i d, i d)$ and $(\alpha, \beta)^{-1}=\left(\alpha^{-1}, \alpha \beta^{-1} \alpha^{-1}\right)$ ).

Proposition 3.2 Let $N \in{ }_{H} \mathcal{Y D}^{H}(\gamma, \delta)$ and $(\alpha, \beta) \in G$. Define ${ }^{(\alpha, \beta)} N=N$ as vector space, with structures

$$
\begin{aligned}
& h \rightarrow n=\gamma^{-1} \beta \gamma \alpha^{-1}(h) \cdot n, \\
& n \mapsto n_{<0>} \otimes n_{<1>}=n_{(0)} \otimes \alpha \beta^{-1}\left(n_{(1)}\right) .
\end{aligned}
$$

Then $^{(\alpha, \beta)} N \in{ }_{H} \mathcal{Y} \mathcal{D}^{H}\left(\alpha \gamma \alpha^{-1}, \alpha \beta^{-1} \delta \gamma^{-1} \beta \gamma \alpha^{-1}\right)={ }_{H} \mathcal{Y} \mathcal{D}^{H}\left((\alpha, \beta) *(\gamma, \delta) *(\alpha, \beta)^{-1}\right)$.

Proof. Obviously ${ }^{(\alpha, \beta)} N$ is a left $H$-module and right $H$-comodule, so we check the compatibility condition. We compute:

$$
\begin{aligned}
(h \rightarrow n)_{<0>} & \otimes(h \rightarrow n)_{<1>} \\
& =\left(\gamma^{-1} \beta \gamma \alpha^{-1}(h) \cdot n\right)_{(0)} \otimes \alpha \beta^{-1}\left(\left(\gamma^{-1} \beta \gamma \alpha^{-1}(h) \cdot n\right)_{(1)}\right) \\
& =\gamma^{-1} \beta \gamma \alpha^{-1}\left(h_{2}\right) \cdot n_{(0)} \otimes \alpha \beta^{-1}\left(\delta \gamma^{-1} \beta \gamma \alpha^{-1}\left(h_{3}\right) n_{(1)} \gamma \gamma^{-1} \beta \gamma \alpha^{-1}\left(S^{-1}\left(h_{1}\right)\right)\right) \\
& =\gamma^{-1} \beta \gamma \alpha^{-1}\left(h_{2}\right) \cdot n_{(0)} \otimes \alpha \beta^{-1} \delta \gamma^{-1} \beta \gamma \alpha^{-1}\left(h_{3}\right) \alpha \beta^{-1}\left(n_{(1)}\right) \alpha \gamma \alpha^{-1}\left(S^{-1}\left(h_{1}\right)\right) \\
& =h_{2} \rightarrow n_{(0)} \otimes \alpha \beta^{-1} \delta \gamma^{-1} \beta \gamma \alpha^{-1}\left(h_{3}\right) n_{<1>} \alpha \gamma \alpha^{-1}\left(S^{-1}\left(h_{1}\right)\right),
\end{aligned}
$$

that is ${ }^{(\alpha, \beta)} N \in{ }_{H} \mathcal{Y} \mathcal{D}^{H}\left(\alpha \gamma \alpha^{-1}, \alpha \beta^{-1} \delta \gamma^{-1} \beta \gamma \alpha^{-1}\right)$.

Remark 3.3 Let $M \in{ }_{H} \mathcal{Y} \mathcal{D}^{H}(\alpha, \beta), N \in{ }_{H} \mathcal{Y} \mathcal{D}^{H}(\gamma, \delta)$ and $(\mu, \nu) \in G$. Then we have

$$
{ }^{(\alpha, \beta) *(\mu, \nu)} N={ }^{(\alpha, \beta)}\left({ }^{(\mu, \nu)} N\right)
$$

as objects in ${ }_{H} \mathcal{Y} \mathcal{D}^{H}\left(\alpha \mu \gamma \mu^{-1} \alpha^{-1}, \alpha \beta^{-1} \mu \nu^{-1} \delta \gamma^{-1} \nu \mu^{-1} \beta \mu \gamma \mu^{-1} \alpha^{-1}\right)$, and

$$
{ }^{(\mu, \nu)}(M \otimes N)={ }^{(\mu, \nu)} M \otimes{ }^{(\mu, \nu)} N
$$

as objects in ${ }_{H} \mathcal{Y} \mathcal{D}^{H}\left(\mu \alpha \gamma \mu^{-1}, \mu \nu^{-1} \delta \gamma^{-1} \beta \alpha^{-1} \nu \alpha \gamma \mu^{-1}\right)$.

Proposition 3.4 Let $M \in{ }_{H} \mathcal{Y} \mathcal{D}^{H}(\alpha, \beta)$ and $N \in{ }_{H} \mathcal{Y D}^{H}(\gamma, \delta)$. Define ${ }^{M} N={ }^{(\alpha, \beta)} N$ as object in ${ }_{H} \mathcal{Y D}^{H}\left((\alpha, \beta) *(\gamma, \delta) *(\alpha, \beta)^{-1}\right)$. Define the map

$$
c_{M, N}: M \otimes N \rightarrow{ }^{M} N \otimes M, \quad c_{M, N}(m \otimes n)=n_{(0)} \otimes \beta^{-1}\left(n_{(1)}\right) \cdot m .
$$

Then $c_{M, N}$ is $H$-linear $H$-colinear and satisfies the conditions (for $P \in{ }_{H} \mathcal{Y D}^{H}(\mu, \nu)$ ):

$$
\begin{aligned}
& c_{M \otimes N, P}=\left(c_{M,{ }^{N} P} \otimes i d_{N}\right) \circ\left(i d_{M} \otimes c_{N, P}\right), \\
& c_{M, N \otimes P}=\left(i d_{M_{N}} \otimes c_{M, P}\right) \circ\left(c_{M, N} \otimes i d_{P}\right) .
\end{aligned}
$$

Moreover, if $M \in{ }_{H} \mathcal{Y} \mathcal{D}^{H}(\alpha, \beta), N \in{ }_{H} \mathcal{Y D}^{H}(\gamma, \delta)$ and $(\mu, \nu) \in G$, then $c_{(\mu, \nu)}{ }_{M,(\mu, \nu)}{ }_{N}=c_{M, N}$. 
Proof. We prove that $c_{M, N}$ is $H$-linear. We compute:

$$
\begin{aligned}
c_{M, N}(h \cdot(m \otimes n)) \\
=c_{M, N}\left(\gamma\left(h_{1}\right) \cdot m \otimes \gamma^{-1} \beta \gamma\left(h_{2}\right) \cdot n\right) \\
=\left(\gamma^{-1} \beta \gamma\left(h_{2}\right) \cdot n\right)_{(0)} \otimes \beta^{-1}\left(\left(\gamma^{-1} \beta \gamma\left(h_{2}\right) \cdot n\right)_{(1)}\right) \gamma\left(h_{1}\right) \cdot m \\
=\gamma^{-1} \beta \gamma\left(h_{2}\right)_{2} \cdot n_{(0)} \otimes \beta^{-1}\left(\delta\left(\gamma^{-1} \beta \gamma\left(h_{2}\right)_{3}\right) n_{(1)} \gamma\left(S^{-1}\left(\left(\gamma^{-1} \beta \gamma\left(h_{2}\right)\right)_{1}\right)\right)\right) \gamma\left(h_{1}\right) \cdot m \\
=\gamma^{-1} \beta \gamma\left(h_{3}\right) \cdot n_{(0)} \otimes \beta^{-1} \delta \gamma^{-1} \beta \gamma\left(h_{4}\right) \beta^{-1}\left(n_{(1)}\right) \gamma\left(S^{-1}\left(h_{2}\right)\right) \gamma\left(h_{1}\right) \cdot m \\
=\gamma^{-1} \beta \gamma\left(h_{1}\right) \cdot n_{(0)} \otimes \beta^{-1} \delta \gamma^{-1} \beta \gamma\left(h_{2}\right) \beta^{-1}\left(n_{(1)}\right) \cdot m, \\
= \\
=c_{M, N}(m \otimes n)\left(n_{(0)} \otimes \beta^{-1}\left(n_{(1)}\right) \cdot m\right) \\
=\alpha\left(h_{1}\right) \rightarrow n_{(0)} \otimes \alpha^{-1} \alpha \beta^{-1} \delta \gamma^{-1} \beta \gamma \alpha^{-1} \alpha\left(h_{2}\right) \beta^{-1}\left(n_{(1)}\right) \cdot m \\
=\gamma^{-1} \beta \gamma \alpha^{-1} \alpha\left(h_{1}\right) \cdot n_{(0)} \otimes \beta^{-1} \delta \gamma^{-1} \beta \gamma\left(h_{2}\right) \beta^{-1}\left(n_{(1)}\right) \cdot m \\
=\gamma^{-1} \beta \gamma\left(h_{1}\right) \cdot n_{(0)} \otimes \beta^{-1} \delta \gamma^{-1} \beta \gamma\left(h_{2}\right) \beta^{-1}\left(n_{(1)}\right) \cdot m,
\end{aligned}
$$

so the two terms are equal. The fact that $c_{M, N}$ is $H$-colinear is similar and left to the reader. We prove now (3.2). First note that, due to Remark 3.3 we have ${ }^{M}\left({ }^{N} P\right)={ }^{M \otimes N} P$ and ${ }^{M}(N \otimes P)={ }^{M} N \otimes{ }^{M} P$. We compute:

$$
\begin{aligned}
\left(c_{M,{ }_{P}} \otimes i d_{N}\right) \circ\left(i d_{M} \otimes c_{N, P}\right)(m \otimes n \otimes p) & =c_{M,{ }_{P}}\left(m \otimes p_{(0)}\right) \otimes \delta^{-1}\left(p_{(1)}\right) \cdot n \\
& =p_{(0)_{<0>}} \otimes \beta^{-1}\left(p_{(0)_{<1>}}\right) \cdot m \otimes \delta^{-1}\left(p_{(1)}\right) \cdot n \\
& =p_{(0)_{(0)}} \otimes \beta^{-1} \gamma \delta^{-1}\left(p_{(0)}\right) \cdot m \otimes \delta^{-1}\left(p_{(1)}\right) \cdot n \\
& =p_{(0)} \otimes \beta^{-1} \gamma \delta^{-1}\left(p_{(1)_{1}}\right) \cdot m \otimes \delta^{-1}\left(p_{(1)_{2}}\right) \cdot n, \\
c_{M \otimes N, P}(m \otimes n \otimes p) & =p_{(0)} \otimes \gamma^{-1} \beta^{-1} \gamma \delta^{-1}\left(p_{(1)}\right) \cdot(m \otimes n) \\
& =p_{(0)} \otimes \gamma \gamma^{-1} \beta^{-1} \gamma \delta^{-1}\left(p_{(1)_{1}}\right) \cdot m \otimes \gamma^{-1} \beta \gamma \gamma^{-1} \beta^{-1} \gamma \delta^{-1}\left(p_{(1)_{2}}\right) \cdot n \\
& =p_{(0)} \otimes \beta^{-1} \gamma \delta^{-1}\left(p_{(1)_{1}}\right) \cdot m \otimes \delta^{-1}\left(p_{(1)_{2}}\right) \cdot n,
\end{aligned}
$$

and we are done. The proof of (3.3) is easier and left to the reader, and similarly the last statement of the Proposition.

Note that $c_{M, N}$ is bijective with inverse $c_{M, N}^{-1}(n \otimes m)=\beta^{-1}\left(S\left(n_{(1)}\right)\right) \cdot m \otimes n_{(0)}$.

We are ready now to introduce the desired braided T-category (we use terminology as in [18; for the subject of Turaev categories, see also the original paper of Turaev [16] and 2], 17]). Define $\mathcal{Y} \mathcal{D}(H)$ as the disjoint union of all ${ }_{H} \mathcal{Y D}^{H}(\alpha, \beta)$, with $(\alpha, \beta) \in G$ (hence the component of the unit is just $\left.{ }_{H} \mathcal{Y} \mathcal{D}^{H}\right)$. If we endow $\mathcal{Y} \mathcal{D}(H)$ with tensor product as in Proposition 3.1 then it becomes a strict monoidal category with unit $k$ as object in ${ }_{H} \mathcal{Y D}^{H}$ (with trivial structures). The group homomorphism $\varphi: G \rightarrow \operatorname{aut}(\mathcal{Y} \mathcal{D}(H)),(\alpha, \beta) \mapsto \varphi_{(\alpha, \beta)}$, is given on components as

$$
\varphi_{(\alpha, \beta)}:{ }_{H} \mathcal{Y} \mathcal{D}^{H}(\gamma, \delta) \rightarrow{ }_{H} \mathcal{Y} \mathcal{D}^{H}\left((\alpha, \beta) *(\gamma, \delta) *(\alpha, \beta)^{-1}\right), \quad \varphi_{(\alpha, \beta)}(N)={ }^{(\alpha, \beta)} N,
$$

and the functor $\varphi_{(\alpha, \beta)}$ acts as identity on morphisms. The braiding in $\mathcal{Y} \mathcal{D}(H)$ is given by the family $\left\{c_{M, N}\right\}$. As a consequence of the above results, we obtain:

Theorem 3.5 $\mathcal{Y} \mathcal{D}(H)$ is a braided T-category over $G$. 
We consider now the problem of existence of left and right dualities.

Proposition 3.6 Let $M \in{ }_{H} \mathcal{Y D}^{H}(\alpha, \beta)$ and assume that $M$ is finite dimensional. Then $M^{*}=$ $\operatorname{Hom}(M, k)$ becomes an object in ${ }_{H} \mathcal{Y D}^{H}\left(\alpha^{-1}, \alpha \beta^{-1} \alpha^{-1}\right)$, with $(h \cdot f)(m)=f\left(\left(\beta^{-1} \alpha^{-1} S(h)\right) \cdot m\right)$ and $f_{(0)}(m) \otimes f_{(1)}=f\left(m_{(0)}\right) \otimes S^{-1}\left(m_{(1)}\right)$. Moreover, the maps $b_{M}: k \rightarrow M \otimes M^{*}, b_{M}(1)=$ $\sum_{i} e_{i} \otimes e^{i}$ (where $\left\{e_{i}\right\}$ and $\left\{e^{i}\right\}$ are dual bases in $M$ and $\left.M^{*}\right)$ and $d_{M}: M^{*} \otimes M \rightarrow k, d_{M}(f \otimes$ $m)=f(m)$, are morphisms in ${ }_{H} \mathcal{Y D}^{H}$ and we have $\left(i d_{M} \otimes d_{M}\right)\left(b_{M} \otimes i d_{M}\right)=i d_{M}$ and $\left(d_{M} \otimes\right.$ $\left.i d_{M^{*}}\right)\left(i d_{M^{*}} \otimes b_{M}\right)=i d_{M^{*}}$.

Proof. We first prove that $M^{*}$ is indeed an object in ${ }_{H} \mathcal{Y} \mathcal{D}^{H}\left(\alpha^{-1}, \alpha \beta^{-1} \alpha^{-1}\right)$. We compute:

$$
\begin{aligned}
& \quad(h \cdot f)_{(0)}(m) \otimes(h \cdot f)_{(1)}=(h \cdot f)\left(m_{(0)}\right) \otimes S^{-1}\left(m_{(1)}\right) \\
& =f\left(\left(\beta^{-1} \alpha^{-1} S\right)(h) \cdot m_{(0)}\right) \otimes S^{-1}\left(m_{(1)}\right), \\
& \left(h_{(2)} \cdot f_{(0)}\right)(m) \otimes\left(\alpha \beta^{-1} \alpha^{-1}\right)\left(h_{(3)}\right) f_{(1)}\left(\alpha^{-1} S^{-1}\right)\left(h_{(1)}\right) \\
& =f_{(0)}\left(\beta^{-1} \alpha^{-1} S\left(h_{(2)}\right) \cdot m\right) \otimes\left(\alpha \beta^{-1} \alpha^{-1}\right)\left(h_{(3)}\right) f_{(1)}\left(\alpha^{-1} S^{-1}\left(h_{(1)}\right)\right) \\
& =f\left(\left(\left(\beta^{-1} \alpha^{-1} S\right)\left(h_{(2)}\right) \cdot m\right)_{(0)}\right) \otimes\left(\alpha \beta^{-1} \alpha^{-1}\left(h_{(3)}\right)\right) \\
& S^{-1}\left(\left(\left(\beta^{-1} \alpha^{-1} S\right)\left(h_{(2)}\right) \cdot m\right)_{(1)}\right)\left(\alpha^{-1} S^{-1}\left(h_{(1)}\right)\right) \\
& =f\left(\left(\beta^{-1} \alpha^{-1} S\right)\left(h_{(3)}\right) \cdot m_{(0)}\right) \otimes\left(\alpha \beta^{-1} \alpha^{-1}\left(h_{(5)}\right)\right) \\
& \quad S^{-1}\left(\left(\alpha^{-1} S\right)\left(h_{(2)}\right) m_{(1)}\left(\alpha \beta^{-1} \alpha^{-1}\right)\left(h_{(4)}\right)\right)\left(\alpha^{-1} S^{-1}\left(h_{(1)}\right)\right) \\
& =f\left(\left(\beta^{-1} \alpha^{-1} S\right)\left(h_{(3)}\right) \cdot m_{(0)}\right) \otimes\left(\alpha \beta^{-1} \alpha^{-1}\right)\left(h_{(5)} S^{-1}\left(h_{(4)}\right)\right) S^{-1}\left(m_{(1)}\right) \alpha^{-1}\left(h_{(2)} S^{-1}\left(h_{(1)}\right)\right) \\
& =f\left(\left(\beta^{-1} \alpha^{-1} S\right)(h) \cdot m_{(0)}\right) \otimes S^{-1}\left(m_{(1)}\right),
\end{aligned}
$$

which means that

$$
(h \cdot f)_{(0)} \otimes(h \cdot f)_{(1)}=\left(h_{(2)} \cdot f_{(0)}\right) \otimes\left(\alpha \beta^{-1} \alpha^{-1}\right)\left(h_{(3)}\right) f_{(1)}\left(\alpha^{-1} S^{-1}\right)\left(h_{(1)}\right), \text { q.e.d. }
$$

On $k$ we have the trivial module and comodule structure, and with these $k \in{ }_{H} \mathcal{Y} \mathcal{D}^{H}$. We want to prove that $b_{M}$ and $d_{M}$ are $H$-module maps. We compute:

$$
\begin{aligned}
\left(h \cdot b_{M}(1)\right)(m)= & \left(h \cdot\left(\sum_{i} e_{i} \otimes e^{i}\right)\right)(m) \\
= & \sum_{i} \alpha^{-1}\left(h_{(1)}\right) \cdot e_{i} \otimes\left(\left(\alpha \beta \alpha^{-1}\right)\left(h_{(2)}\right) \cdot e^{i}\right)(m) \\
= & \alpha^{-1}\left(h_{(1)}\right) \cdot e_{i} \otimes e^{i}\left(\left(\beta^{-1} \alpha^{-1} S \alpha \beta \alpha^{-1}\right)\left(h_{(2)}\right) \cdot m\right) \\
= & \sum_{i} \alpha^{-1}\left(h_{(1)}\right) \cdot e_{i} \otimes e^{i}\left(\left(\alpha^{-1} S\right)\left(h_{(2)}\right) \cdot m\right) \\
= & \alpha^{-1}\left(h_{(1)} S\left(h_{(2)}\right)\right) \cdot m \\
= & \varepsilon(h) \sum_{i} e_{i} \otimes e^{i}(m) \\
= & \left(\varepsilon(h) b_{M}(1)\right)(m), \\
\quad= & d_{M}\left(\alpha\left(h_{(1)}\right) \cdot f \otimes \beta^{-1}\left(h_{(2)}\right) \cdot m\right) \\
= & \left(\alpha\left(h_{(1)}\right) \cdot f\right)\left(\beta^{-1}\left(h_{(2)}\right) \cdot m\right) \\
\quad= & f\left(\left(\beta^{-1} \alpha^{-1} S \alpha\left(h_{(1)}\right)\right) \beta^{-1}\left(h_{(2)}\right) \cdot m\right) \\
& =f\left(\beta^{-1}\left(S\left(h_{(1)}\right) h_{(2)}\right) \cdot m\right) \\
& =\varepsilon(h) d_{M}(f \otimes m) .
\end{aligned}
$$


Also they are $H$-comodule maps:

$$
\begin{aligned}
\left(\left(b_{M}(1)\right)_{(0)} \otimes\left(b_{M}(1)\right)_{(1)}\right)(m) & =\sum_{i}\left(e_{i}\right)_{(0)} \otimes\left(e^{i}\right)_{(0)}(m) \otimes\left(e^{i}\right)_{(1)}\left(e_{i}\right)_{(1)} \\
& =\sum_{i}\left(e_{i}\right)_{(0)} \otimes\left(e^{i}\right)\left(m_{(0)}\right) \otimes S^{-1}\left(m_{(1)}\right)\left(e_{i}\right)_{(1)} \\
& =m_{(0)} \otimes S^{-1}\left(m_{(1)_{2}}\right) m_{(1)_{1}} \\
& =\left(b_{M}(1) \otimes 1\right)(m), \\
& \\
d_{M}\left((f \otimes m)_{(0)}\right) \otimes(f \otimes m)_{(1)} & =f_{(0)}\left(m_{(0)}\right) \otimes m_{(1)} f_{(1)} \\
& =f\left(m_{(0)}\right) \otimes m_{(1)_{2}} S^{-1}\left(m_{(1)_{1}}\right) \\
& =d_{M}(f \otimes m) \otimes 1 .
\end{aligned}
$$

Finally, the last two identities $\left(i d_{M} \otimes d_{M}\right)\left(b_{M} \otimes i d_{M}\right)=i d_{M}$ and $\left(d_{M} \otimes i d_{M^{*}}\right)\left(i d_{M^{*}} \otimes b_{M}\right)=i d_{M^{*}}$ are trivial.

Similarly, one can prove:

Proposition 3.7 Let $M \in{ }_{H} \mathcal{Y D}^{H}(\alpha, \beta)$ and assume that $M$ is finite dimensional. Then ${ }^{*} M=$ $\operatorname{Hom}(M, k)$ becomes an object in ${ }_{H} \mathcal{Y D}^{H}\left(\alpha^{-1}, \alpha \beta^{-1} \alpha^{-1}\right)$, with $(h \cdot f)(m)=f\left(\left(\alpha^{-1} \beta^{-1} S^{-1}(h)\right)\right.$. $m)$ and $f_{(0)}(m) \otimes f_{(1)}=f\left(m_{(0)}\right) \otimes S\left(m_{(1)}\right)$. Moreover, the maps $b_{M}: k \rightarrow{ }^{*} M \otimes M, b_{M}(1)=$ $\sum_{i} e^{i} \otimes e_{i}$ and $d_{M}: M \otimes{ }^{*} M \rightarrow k, d_{M}(m \otimes f)=f(m)$, are morphisms in ${ }_{H} \mathcal{Y D}^{H}$ and we have $\left(d_{M} \otimes i d_{M}\right)\left(i d_{M} \otimes b_{M}\right)=i d_{M}$ and $\left(i d{ }^{*} M \otimes d_{M}\right)\left(b_{M} \otimes i d *_{M}\right)=i d *_{M}$.

Consequently, if we consider $\mathcal{Y} \mathcal{D}(H)_{f d}$, the subcategory of $\mathcal{Y} \mathcal{D}(H)$ consisting of finite dimensional objects, we obtain:

Theorem 3.8 $\mathcal{Y} \mathcal{D}(H)_{f d}$ is a braided T-category with left and right dualities over $G$, the left (respectively right) duals being given as in Proposition 3.6 (respectively Proposition 3.7).

Assume now that $H$ is finite dimensional. We will construct a quasitriangular T-coalgebra over $G$, denoted by $D T(H)$, with the property that the T-category $\operatorname{Rep}(D T(H))$ of representations of $D T(H)$ is isomorphic to $\mathcal{Y} \mathcal{D}(H)$ as braided T-categories.

For $(\alpha, \beta) \in G$, the $(\alpha, \beta)$-component $D T(H)_{(\alpha, \beta)}$ will be the diagonal crossed product algebra $H^{*} \bowtie H(\alpha, \beta)$. Define

$$
\begin{aligned}
& \Delta_{(\alpha, \beta),(\gamma, \delta)}: H^{*} \bowtie H((\alpha, \beta) *(\gamma, \delta)) \rightarrow\left(H^{*} \bowtie H(\alpha, \beta)\right) \otimes\left(H^{*} \bowtie H(\gamma, \delta)\right), \\
& \Delta_{(\alpha, \beta),(\gamma, \delta)}(p \bowtie h)=\left(p_{2} \bowtie \gamma\left(h_{1}\right)\right) \otimes\left(p_{1} \bowtie \gamma^{-1} \beta \gamma\left(h_{2}\right)\right) .
\end{aligned}
$$

One can check, by direct computation, that these maps are algebra maps, satisfying the necessary coassociativity conditions.

The counit $\varepsilon$ is just the counit of $D T(H)_{(i d, i d)}=D(H)$, the Drinfeld double of $H$.

For $(\alpha, \beta),(\gamma, \delta) \in G$, define now

$$
\begin{aligned}
& \varphi_{(\alpha, \beta)}^{(\gamma, \delta)}: H^{*} \bowtie H(\gamma, \delta) \rightarrow H^{*} \bowtie H\left((\alpha, \beta) *(\gamma, \delta) *(\alpha, \beta)^{-1}\right), \\
& \varphi_{(\alpha, \beta)}^{(\gamma, \delta)}(p \bowtie h)=p \circ \beta \alpha^{-1} \bowtie \alpha \gamma^{-1} \beta^{-1} \gamma(h) .
\end{aligned}
$$


Then one can check by direct computation that these are algebra isomorphisms giving a conjugation (that is they are multiplicative and compatible with the comultiplications and the counit). The antipode is given, for $(\alpha, \beta) \in G$, by

$$
\begin{aligned}
& S_{(\alpha, \beta)}: H^{*} \bowtie H(\alpha, \beta) \rightarrow H^{*} \bowtie H\left((\alpha, \beta)^{-1}\right), \\
& S_{(\alpha, \beta)}(p \bowtie h)=(\varepsilon \bowtie \alpha \beta(S(h))) \cdot\left(S^{*-1}(p) \bowtie 1\right),
\end{aligned}
$$

where the multiplication - in the right hand side is made in $H^{*} \bowtie H\left((\alpha, \beta)^{-1}\right)$.

Finally, the universal $R$-matrix is given by

$$
R_{(\alpha, \beta),(\gamma, \delta)}=\sum_{i}\left(\varepsilon \bowtie \beta^{-1}\left(e_{i}\right)\right) \otimes\left(e^{i} \bowtie 1\right) \in\left(H^{*} \bowtie H(\alpha, \beta)\right) \otimes\left(H^{*} \bowtie H(\gamma, \delta)\right),
$$

for all $(\alpha, \beta),(\gamma, \delta) \in G$, where $\left\{e_{i}\right\},\left\{e^{i}\right\}$ are dual bases in $H$ and $H^{*}$.

Thus, we have obtained:

Theorem 3.9 DT(H) is a quasitriangular T-coalgebra over $G$, with structure as above.

Moreover, the structure of $D T(H)$ was constructed in such a way that, via the isomorphisms $H_{H^{*} \bowtie H(\alpha, \beta)} \mathcal{M} \simeq{ }_{H} \mathcal{Y} \mathcal{D}^{H}(\alpha, \beta)$ from Section [2] we obtain:

Theorem 3.10 $\operatorname{Rep}(D T(H))$ and $\mathcal{Y} \mathcal{D}(H)$ are isomorphic as braided T-categories over $G$.

Remark 3.11 From $\mathcal{Y} \mathcal{D}(H)$ (respectively $D T(H)$ ) we can obtain, by pull-back along the group morphism $\operatorname{Aut}_{\text {Hopf }}(H) \rightarrow G, \alpha \mapsto(\alpha, i d)$, a braided T-category (respectively a quasitriangular T-coalgebra) over $A u t_{\text {Hopf }}(H)$. If $\pi$ is a group together with a group morphism $\pi \rightarrow$ $\operatorname{Aut}_{\text {Hopf }}(H)$, by pull-back along it we obtain a braided T-category (respectively a quasitriangular T-coalgebra) over $\pi$. Quasitriangular T-coalgebras over such $\pi$ have been studied by Virelizier in [17.

\section{An isomorphism of categories ${ }_{H} \mathcal{Y} \mathcal{D}^{H}(\alpha, \beta) \simeq{ }_{H} \mathcal{Y} \mathcal{D}^{H}$ in the presence of a pair in involution}

The aim of this section is to prove the following result.

Theorem 4.1 Let $\alpha, \beta \in$ Aut $_{\text {Hopf }}(H)$ and assume that there exists $(f, g)$ a pair in involution corresponding to $(\alpha, \beta)$. Then the categories ${ }_{H} \mathcal{Y D}^{H}(\alpha, \beta)$ and ${ }_{H} \mathcal{Y D}^{H}$ are isomorphic.

A pair of inverse functors $(F, G)$ is given as follows. If $M \in{ }_{H} \mathcal{Y} \mathcal{D}^{H}(\alpha, \beta)$, then $F(M) \in{ }_{H} \mathcal{Y} \mathcal{D}^{H}$, where $F(M)=M$ as vector space, with structures

$$
\begin{aligned}
& h \rightarrow m=f\left(\beta^{-1}\left(S\left(h_{1}\right)\right)\right) \beta^{-1}\left(h_{2}\right) \cdot m, \\
& m \mapsto m_{<0>} \otimes m_{<1>}=m_{(0)} \otimes m_{(1)} g^{-1} .
\end{aligned}
$$

If $N \in{ }_{H} \mathcal{Y} \mathcal{D}^{H}$, then $G(N) \in{ }_{H} \mathcal{Y D}^{H}(\alpha, \beta)$, where $G(N)=N$ as vector space, with structures

$$
\begin{aligned}
& h \rightarrow n=f\left(h_{1}\right) \beta\left(h_{2}\right) \cdot n, \\
& n \mapsto n^{(0)} \otimes n^{(1)}=n_{(0)} \otimes n_{(1)} g .
\end{aligned}
$$

Both $F$ and $G$ act as identities on morphisms. 
Proof. One checks, by direct computation, that $F$ and $G$ are functors, inverse to each other. Alternatively, we can give a very short proof using results from the previous section. By Example 2.7 we have ${ }_{f} k^{g} \in{ }_{H} \mathcal{Y} \mathcal{D}^{H}(\alpha, \beta)$. By Proposition 3.6. we get $\left({ }_{f} k^{g}\right)^{*} \in{ }_{H} \mathcal{Y} \mathcal{D}^{H}\left((\alpha, \beta)^{-1}\right)$. Then, one can check that actually $F(M)=\left({ }_{f} k^{g}\right)^{*} \otimes M \in{ }_{H} \mathcal{Y} \mathcal{D}^{H}$ and $G(N)=\left({ }_{f} k^{g}\right) \otimes N \in$ ${ }_{H} \mathcal{Y} \mathcal{D}^{H}(\alpha, \beta)$. Also, one can see that $\left({ }_{f} k^{g}\right)^{*} \otimes{ }_{f} k^{g}={ }_{f} k^{g} \otimes\left({ }_{f} k^{g}\right)^{*}=k$ as objects in ${ }_{H} \mathcal{Y} \mathcal{D}^{H}$, hence $F \circ G=G \circ F=i d$, using the associativity of the tensor product.

As we have noticed before, for any $\alpha \in \operatorname{Aut}_{\text {Hopf }}(H)$ we have that $(\varepsilon, 1)$ is a pair in involution corresponding to $(\alpha, \alpha)$, hence we obtain:

Corollary $4.2{ }_{H} \mathcal{Y} \mathcal{D}^{H}(\alpha, \alpha) \simeq{ }_{H} \mathcal{Y} \mathcal{D}^{H}$.

Also, as a consequence of the theorem, we obtain the following result (a right-left version was given in [14]), which might be useful for the aria of applicability of anti-Yetter-Drinfeld modules:

Corollary 4.3 Assume that there exists a pair in involution $(f, g)$ corresponding to $\left(S^{2}, i d\right)$. Then the category ${ }_{H} \mathcal{Y D}^{H}\left(S^{2}, i d\right)$ of anti-Yetter-Drinfeld modules is isomorphic to ${ }_{H} \mathcal{Y D}^{H}$, and any anti-Yetter-Drinfeld module can be written as a tensor product ${ }_{f} k^{g} \otimes N$, with $N \in{ }_{H} \mathcal{Y D}^{H}$.

Let again $\alpha, \beta \in \operatorname{Aut}_{\text {Hopf }}(H)$ such that there exists $(f, g)$ a pair in involution corresponding to $(\alpha, \beta)$, and assume that $H$ is finite dimensional. Then we know that ${ }_{H} \mathcal{Y} \mathcal{D}^{H}(\alpha, \beta) \simeq H^{*} \bowtie H(\alpha, \beta) \mathcal{M}$, ${ }_{H} \mathcal{Y} \mathcal{D}^{H} \simeq{ }_{D(H)} \mathcal{M}$, and the isomorphism ${ }_{H} \mathcal{Y D}^{H}(\alpha, \beta) \simeq{ }_{H} \mathcal{Y} \mathcal{D}^{H}$ constructed in the theorem is induced by an algebra isomorphism between $H^{*} \bowtie H(\alpha, \beta)$ and $D(H)$, given by

$$
\begin{array}{ll}
D(H) \rightarrow H^{*} \bowtie H(\alpha, \beta), & p \otimes h \mapsto g^{-1} \rightarrow p \bowtie f\left(\beta^{-1}\left(S\left(h_{1}\right)\right)\right) \beta^{-1}\left(h_{2}\right), \\
H^{*} \bowtie H(\alpha, \beta) \rightarrow D(H), & p \bowtie h \mapsto g \rightarrow p \otimes f\left(h_{1}\right) \beta\left(h_{2}\right) .
\end{array}
$$

\section{References}

[1] D. Bulacu, F. Panaite, F. Van Oystaeyen, Generalized diagonal crossed products and smash products for (quasi) Hopf algebras. Applications, in preparation.

[2] S. Caenepeel, M. De Lombaerde, A categorical approach to Turaev's Hopf group-coalgebras, arXiv:math.QA/0409600.

[3] S. Caenepeel, G. Militaru, S. Zhu, "Frobenius and separable functors for generalized module categories and nonlinear equations", Lecture Notes in Mathematics 1787, Springer Verlag, Berlin, 2002.

[4] S. Caenepeel, F. Van Oystaeyen, Y. Zhang, The Brauer group of Yetter-Drinfeld module algebras, Trans. Amer. Math. Soc. 349 (1997), 3737-3771.

[5] A. Connes, H. Moscovici, Hopf algebras, cyclic cohomology and the transverse index theorem, Comm. Math. Phys. 198 (1998), 199-264.

[6] A. Connes, H. Moscovici, Cyclic cohomology and Hopf algebra symmetry, Lett. Math. Phys. 52 (2000), 1-28.

[7] P. M. Hajac, M. Khalkhali, B. Rangipour, Y. Sommerhauser, Stable anti-Yetter-Drinfeld modules, C. R. Math. Acad. Sci. Paris 338 (2004), 587-590. 
[8] P. M. Hajac, M. Khalkhali, B. Rangipour, Y. Sommerhauser, Hopf-cyclic homology and cohomology with coefficients, C. R. Math. Acad. Sci. Paris 338 (2004), 667-672.

[9] F. Hausser and F. Nill, Diagonal crossed products by duals of quasi-quantum groups, Rev. Math. Phys. 11 (1999), 553-629.

[10] P. Jara, D. Ştefan, Cyclic homology of Hopf Galois extensions and Hopf algebras, arXiv:math.KT/0307099

[11] C. Kassel, "Quantum groups", Graduate Texts in Mathematics 155, Springer Verlag, Berlin, 1995.

[12] S. Majid, "Foundations of quantum group theory", Cambridge Univ. Press, 1995.

[13] S. Montgomery, "Hopf algebras and their actions on rings", CBMS Regional Conference Series, Vol. 82, Amer. Math. Soc., Providence, RI, 1993.

[14] M. D. Staic, A note on anti-Yetter-Drinfeld modules, preprint 2004.

[15] M. E. Sweedler, "Hopf algebras", Benjamin, New York, 1969.

[16] V. Turaev, Homotopy field theory in dimension 3 and crossed group-categories, arXiv:math.GT/0005291

[17] A. Virelizier, Graded quantum groups, arXiv:math.QA/0312330.

[18] M. Zunino, Yetter-Drinfeld modules for crossed structures, J. Pure Appl. Algebra 193 (2004), 313-343. 